\title{
FMCH (Foederatio Medicorum Chirurgicorum Helvetica) gegründet
}

Pressestelle FMCH

Am Samstag, 18. September 2004, haben in Bern 12 chirurgische Fachgesellschaften den Schweizerischen Verband chirurgisch tätiger Ärzte gegründet und ihm den Namen FMCH gegeben. Die Statuten und das erste Budget der FMCH wurden von den vollzählig anwesenden Präsidenten der involvierten Fachgesellschaften genehmigt. Anlässlich der gleichen Sitzung wurde auch bereits der Vorstand gewählt. Zum ersten Präsidenten der FMCH wurde Prof. Dr. Urban Laffer, Chefarzt der Chirurgischen Klinik am Spitalzentrum Biel, zum Vizepräsidenten Dr. Thomas Egloff, Wirbelsäulenchirurg an der Hirslanden-Klinik Birshof in Basel, gewählt.
Bis vor kurzem wurden die chirurgisch tätigen Ärzte der Schweiz durch zwei Verbände vertreten. Es war dies einerseits die UNION chirurgischer Fachgesellschaften, die sich vor allem mit den Fragen der chirurgischen Weiter- und Fortbildung beschäftigte. Andererseits die FMS (Federatio Medicorum scrutantium), die 1999 gegründet wurde, um die Interessen der chirurgisch und invasiv tätigen Ärzte im Rahmen der TARMED-Verhandlungen zu vertreten. Gerade die Verhandlungen um das neue Tarifwesen zeigten jedoch nachhaltig auf, dass die chirurgischen Fachgesellschaften zwar einen qualitativ hochstehenden und unverzichtbaren Beitrag zum Gesundheitswesen der Schweiz leisten, innerhalb der Ärzteschaft - bedingt durch die jetzige Struktur der FMH - aber nicht über den entsprechenden Einfluss und das notwendige Mitbestimmungsrecht verfügen. Dies führte dazu, dass UNION und FMS Anfang 2003 eine Taskforce bildeten mit dem Ziel, die Grundlagen für einen schlagkräftigen Dachverband der chirurgischen Fachgesellschaften der Schweiz zu erarbeiten. Nach monatelanger, intensiver Arbeit konnten die Vorarbeiten abgeschlossen und der neue Dachverband am 18. September 2004 in Bern gegründet werden.

Der neue Dachverband nennt sich FMCH (Foederatio Medicorum Chirurgicorum Helvetica), folgende Fachgesellschaften sind darin vereinigt:

Schweizerische Gesellschaft für:

- Anästhesiologie und Reanimation (SGAR);

- Chirurgie (SGC);

- Handchirurgie (SGH);

- Kiefer- und Gesichtschirurgie (SGKG);

- Kinderchirurgie (SGKC);

- Neurochirurgie (SGNC);

- Ophthalmologie (SOG);
- Orthopädie und Traumatologie des Bewegungsapparates (SGO);

- Oto-Rhino-Laryngologie, Hals- und Gesichtschirurgie (SGORL);

- Plastische-Rekonstruktive und Ästhetische Chirurgie (SGPRAC);

- Thorax-, Herz- und Gefässchirurgie (SGTHG);

- Urologie (SGU).

Damit umfasst die Basis des neuen Verbandes mehr als 3400 Mitglieder.

Die Ziele der FMCH sind:

- Koordination der chirurgischen Weiter- und Fortbildung;

- Koordination der chirurgischen Qualitätssicherung und des Fehlermanagements;

- Koordination der Tarifangelegenheiten;

- Koordination von Ethik und Recht;

- Schaffen von angemessenen Rahmenbedingungen für die optimale Ausübung der chirurgischen Tätigkeit in der freien Praxis und im Spital;

- Sicherstellen einer starken Vertretung gegenüber FMH, Behörden, Versicherern und weiteren Organisationen;

- Pflegen einer standesgemässen Kommunikation unter den Fachgesellschaften und Sicherstellen der Kommunikation nach aussen (Öffentlichkeitsarbeiten) in berufspolitischen und gesetzgeberischen Belangen;

- Fördern und aktives Einbeziehen des chirurgischen Nachwuchses;

- Fördern der Qualität nach den Prinzipien von Wirksamkeit, Wirtschaftlichkeit und Zweckmässigkeit;

- Wahren der berufspolitischen und wirtschaftlichen Interessen der Mitglieder;

- Optimieren der Ressourcennutzung für die Fachgesellschaften;

- Vermitteln von Dienstleistungen in beruflichen, rechtlichen und wirtschaftlichen Belangen;

- Pflegen und Fördern von Beziehungen zu ärztlichen Organisationen im In- und Ausland.

Oberstes Organ der FMCH ist die Delegiertenversammlung, welcher die Präsidenten sowie je ein Delegierter der Fachgesellschaften an- 
gehören. Exekutivorgan ist der Vorstand zusammen mit dem Generalsekretariat und der Geschäftsstelle.

Anlässlich der Gründungsversammlung wurde der Vorstand sowie Präsident und Vizepräsident der FMCH gewählt. Zum ersten Präsidenten wählte die Versammlung Prof. Dr. Urban Laffer. Urban Laffer war von 2002 bis 2004 Präsident der Schweizerischen Gesellschaft für Chirurgie (SGC) und ist Chefarzt der Chirurgischen Klinik am Spitalzentrum Biel. Zum Vizepräsidenten wählte die Gründungsversammlung Dr. Thomas Egloff. Thomas Egloff war Präsident der Taskforce, welche die Vorarbeiten zur Gründung der FMCH leistete und ist Wirbelsäulenchirurg an der Hirslanden-Klinik Birshof in Basel. Dem Vorstand gehören weiter an: Dr. Josef Brandenberg, Luzern; Prof. Michel Dutoit, Lausanne; Dr. Thomas Kehl, Davos; Prof. Othmar Schöb, Zürich; PD Dr. Thomas Schnider, St. Gallen; Dr. Guido Schüpfer, Luzern; Prof. Markus Wolfensberger, Basel.

Unmittelbar nach der Gründungsversammlung hat der FMCH-Vorstand erstmals getagt und dabei folgende prioritären Aufgaben festgelegt:

_ Zuteilung der verschiedenen Ressorts;
- Einflussnahme in die Strukturreform der $\mathrm{FMH}$;

- Aufbau des Geschäftsstelle, Ausschreibung und Wahl eines/einer Generalsekretärs/Generalsekretärin;

- Kontaktaufnahme mit der Schweizerischen Gesellschaft für Gynäkologie und Geburtshilfe (SGGG), die der FMCH noch nicht beigetreten sind;

- Haftpflichtversicherung für chirurgisch tätige Ärzte.

Der Vorstand der FMCH ist sich bewusst, dass die unmittelbare und weitere Zukunft eine gewaltige Herausforderung bedeutet, die das Engagement jedes einzelnen nachhaltig fordert. Enthusiasmus und Bereitschaft, die bevorstehenden Aufgaben anzupacken, sind vorhanden. Es ist dem Vorstand aber auch klar, dass es nicht möglich sein wird - und auch wenig vernünftig wäre - alle hochstehenden Ziele des neuen Verbandes auf einmal und sofort $\mathrm{zu}$ bewältigen. In diesem Sinne wird er sich vorerst auf die wichtigsten und dringend anstehenden Anliegen konzentrieren, um dann auch die mittel- und langfristigen Aufgaben anpacken zu können.

\section{Fondation de la FMCH (Foederatio Medicorum Chirurgicorum Helvetica)}

Service média FMCH

Samedi 18 septembre 2004, douze sociétés de chirurgiens ont fondé à Berne la Fédération suisse des chirurgiens et l'ont nommée FMCH. Les statuts et le premier budget en ont été approuvés par l'assemblée, au grand complet, des présidents des sociétés concernées. Le comité directeur a été élu au cours de la même réunion. La première présidence a été confiée au Prof. Urban Laffer, médecin-chef responsable de la clinique chirurgicale du Centre hospitalier de Bienne, et la vice-présidence au Dr Thomas Egloff, spécialiste de chirurgie de la colonne vertébrale à la Clinique Hirslanden Birshof de Bâle.

Correspondance:

Service média FMCH

Geri Staudenmann

Haldenstrasse 19 a

CH-2502 Biel/Bienne
Jusque récemment, les chirurgiens suisses étaient représentés par deux fédérations distinctes. Il s'agissait d'une part de l'Union suisse des sociétés chirurgicales, active essentiellement dans le domaine du perfectionnement professionnel et de la formation continue, et d'autre part de la FMS (Foederatio Medicorum Scrutantium), fon- dée en 1999 avec pour but de représenter les préoccupations des médecins pratiquant la chirurgie et les traitements invasifs dans le cadre des négociations TARMED. Cependant, ces négociations tarifaires mirent précisément en lumière le fait que les sociétés de chirurgiens, bien qu'elles fournissent une contribution précieuse et indispensable au secteur de la santé en Suisse, ne disposent, au sein du corps médical - en raison des structures actuelles de la FMH -, ni d'une influence correspondante ni du droit de participation approprié. C'est pourquoi l'Union et la FMS créèrent, au début de 2003, un groupe de travail chargé d'élaborer les bases d'une fédération faîtière des sociétés de chirurgiens suisses. Après plusieurs mois de travaux préparatifs intensifs, la nouvelle fédération put être fondée, le 18 septembre 2004 à Berne. 
La nouvelle fédération se nomme FMCH (Foederatio Medicorum Chirurgicorum Helvetica) et regroupe les sociétés suivantes:

Société suisse:

- d'anesthésiologie et de réanimation (SSAR);

- de chirurgie (SSC);

- de chirurgie de la main (SSCM);

- de chirurgie maxillo-faciale (SSCMF);

- de chirurgie pédiatrique (SSCP);

- de neurochirurgie;

- d'ophtalmologie (SOS);

- d'orthopédie (SSO);

- d'oto-rhino-laryngologie et de chirurgie cervico-faciale;

- de chirurgie plastique, reconstructive et esthétique (SSCPRE);

- de chirurgie thoracique et cardiovasculaire;

- d'urologie.

La nouvelle fédération regroupe ainsi plus de 3400 membres.

La FMCH poursuit les objectifs suivants:

- coordonner le perfectionnement professionnel et la formation continue en chirurgie;

- coordonner la gestion de la qualité et des erreurs de traitement en chirurgie;

- coordonner les questions tarifaires;

- coordonner les questions éthiques et légales;

- établir des conditions cadres appropriées pour l'exercice optimal des activités chirurgicales en cabinet et en clinique;

- assurer une représentation solide et cohérente face à la FMH, aux autorités, aux assureurs et autres organisations;

- maintenir des échanges adéquats entre les sociétés médicales et assurer la communication vers l'extérieur (relations publiques) dans les questions d'ordre politique et législatif;

- favoriser et impliquer activement la relève dans la profession de chirurgien;

- encourager la qualité en respectant les principes d'efficacité, de caractère économique et de valeur thérapeutique;

- préserver les intérêts professionnels et économiques des membres;

- optimiser l'exploitation des ressources dont disposent les sociétés;

- fournir des prestations de services aux niveaux professionnel, légal et économique;

- entretenir et favoriser les relations avec les organisations médicales suisses et étrangères.

L'organe suprême de la FMCH est l'assemblée des délégués, laquelle réunit le président ainsi qu'un délégué de chacune des sociétés médicales. L'or- gane exécutif en est le comité directeur, complété par le secrétaire général et le secrétariat.

$\mathrm{Au}$ cours de l'assemblée constitutive, les membres du comité directeur, le président et le vice-président de la FMCH ont été élus par les délégués. L'assemblée a désigné le Prof. Urban Laffer comme premier président de la Fédération. Urban Laffer présida la Société suisse de chirurgie (SSC) de 2002 à 2004; il exerce la fonction de médecin-chef responsable de la clinique chirurgicale du Centre hospitalier de Bienne. L'assemblée des délégués élut le Dr Thomas Egloff au poste de vice-président. Thomas Egloff présida le groupe de travail chargé de préparer la fondation de la FMCH; il est spécialiste de chirurgie de la colonne vertébrale à la clinique Hirslanden Birshof de Bâle. Ont également été élus au sein du comité directeur: Dr Josef Brandenberg, Lucerne; Prof. Michel Dutoit, Lausanne; Dr Thomas Kehl, Davos; Prof. Othmar Schöb, Zurich; PD Dr Thomas Schnider, Saint-Gall; Dr Guido Schüpfer, Lucerne; Prof. Markus Wolfensberger, Bâle.

Immédiatement après l'assemblée constitutive, le comité directeur de la FMCH a tenu sa première session et, dans ce cadre, a fixé les tâches prioritaires suivantes:

- attribution des différents secteurs de responsabilité;

- implication dans la réforme des structures de la FMH;

- création du secrétariat, mise au concours du poste de secrétaire général/e et désignation d'un/e responsable;

- prise de contact avec la Société suisse de gynécologie et d'obstétrique (SSGO), qui n'a pas encore rejoint la $\mathrm{FMCH}$;

- assurance responsabilité civile pour les médecins pratiquant la chirurgie.

Les membres du comité directeur de la FMCH sont bien conscients que l'avenir leur réserve, à court et moyen termes, des défis considérables, qui mettront à l'épreuve l'engagement de chacun d'entre eux. Ils manifestent à cet égard un grand enthousiasme et sont fermement décidés à maîtriser les tâches qui les attendent. Mais ils savent aussi qu'il ne sera ni possible ni raisonnable d'atteindre tous les ambitieux objectifs de la fédération en même temps et immédiatement. Ils vont donc d'abord concentrer leurs efforts sur les questions les plus importantes et les plus urgentes avant de passer aux projets concernant les moyen et long termes. 